\title{
Hydrogeophysical data integration through Bayesian Sequential Simulation with log-linear pooling
}

\author{
Raphaël Nussbaumer ${ }^{\oplus},{ }^{1}$ Grégoire Mariethoz ${ }^{\oplus}, 2$ Erwan Gloaguen ${ }^{\oplus 3}$ and \\ Klaus Holliger ${ }^{\oplus 1,4}$ \\ ${ }^{1}$ Institute of Earth Sciences, University of Lausanne, 1015 Lausanne, Switzerland.E-mail: raphael.nussbaumer@unil.ch \\ ${ }^{2}$ Institute of Earth Surface Dynamics, University of Lausanne, 1015 Lausanne, Switzerland \\ ${ }^{3}$ INRS, ETE, Quebec G1K 9A9, Quebec City, Canada \\ ${ }^{4}$ School of Earth Sciences, Zhejiang University, 310027 Hangzhou, China
}

Accepted 2020 February 4. Received 2020 January 31; in original form 2019 August 29

\begin{abstract}
S UMMAR Y
Bayesian sequential simulation (BSS) is a geostastistical technique, which uses a secondary variable to guide the stochastic simulation of a primary variable. As such, BSS has proven significant promise for the integration of disparate hydrogeophysical data sets characterized by vastly differing spatial coverage and resolution of the primary and secondary variables. An inherent limitation of BSS is its tendency to underestimate the variance of the simulated fields due to the smooth nature of the secondary variable. Indeed, in its classical form, the method is unable to account for this smoothness because it assumes independence of the secondary variable with regard to neighbouring values of the primary variable. To overcome this limitation, we have modified the Bayesian updating with a log-linear pooling approach, which allows us to account for the inherent interdependence between the primary and the secondary variables by adding exponential weights to the corresponding probabilities. The proposed method is tested on a pertinent synthetic hydrogeophysical data set consisting of surface-based electrical resistivity tomography (ERT) data and local borehole measurements of the hydraulic conductivity. Our results show that, compared to classical BSS, the proposed log-linear pooling method using equal constant weights for the primary and secondary variables enhances the reproduction of the spatial statistics of the stochastic realizations, while maintaining a faithful correspondence with the geophysical data. Significant additional improvements can be achieved by optimizing the choice of these constant weights. We also explore a dynamic adaptation of the weights during the course of the simulation process, which provides valuable insights into the optimal parametrization of the proposed log-linear pooling approach. The results corroborate the strategy of selectively emphasizing the probabilities of the secondary and primary variables at the very beginning and for the remainder of the simulation process, respectively.
\end{abstract}

Key words: Hydrogeophysics; Electrical resistivity tomography (ERT); Statistical methods.

\section{INTRODUCTION}

Adequate knowledge of the hydraulic conductivity distribution within an aquifer is essential for the development of effective groundwater management and remediation strategies. Indeed, the hydraulic conductivity is a critical parameter for hydrological modelling as its spatial distribution determines the flow and transport characteristics of the studied subsurface regions. Corresponding measurements are performed at different scales: core analyses and slug tests provide information with regard to the small-scale heterogeneity, while well tests estimate averages over larger volumes. The corresponding gaps in terms of resolution and coverage can be bridged by specifically targeted geophysical measurements (e.g. Rubin \& Hubbard 2005). The associated field of research is now generally referred to as hydrogeophysics. Arguably, one of the most effective ways to address this problem is through the quantitative integration of geophysical and hydraulic data (e.g. Hyndman \& Gorelick 1996; Dafflon et al. 2009).

Geostatistics offers a toolbox of methods to stochastically populate a grid while respecting a given spatial model as well as incorporating available observed data. Over the years, many geostatistical simulation methods have been developed, such as sequential Gaussian simulation (SGS; e.g. Journel 1989; Deutsch \& Journel 1992), turning bands (e.g. Journel 1974), truncated pluri-Gaussian simulations (Mariethoz et al. 
2009a), simulated annealing (e.g. Deutsch \& Wen 2000) and multipoint geostatistics (e.g. Guardiano \& Srivastava 1993). While such methods are suitable for the simulation of heterogeneous hydraulic conductivity fields, the addition of geophysical data makes them potentially more powerful but also methodologically more complex. Geophysical data, which are generally treated as secondary variables in geostatistical simulations, tend to be available over the entire domain, albeit at a much coarser resolution than the primary variable of interest. Such situations are traditionally addressed through cosimulation (e.g. Gómez-Hernández \& Journel 1993; Verly 1993) or collocated cosimulation assuming a so-called type 2 Markov model (MM2; e.g. Xu et al. 1992; Chilès \& Delfiner 1999). Yet, these methods rely on the inherent assumption of a linear relationship between primary and secondary variables, which is often deemed to be unrealistic (e.g. Gómez-Hernández \& Wen 1998; Zinn \& Harvey 2003).

Bayesian sequential simulation (BSS; Doyen \& Boer 1996) provides an attractive alternative to collocated cosimulation as it does not require a linear or otherwise specific parametric relationship between the primary and the secondary variables. The method is based on SGS, with the addition that the conditional distribution estimated by kriging is updated by the joint distribution of the primary and secondary variables in a Bayesian framework using the value of the collocated secondary variable. While standard cosimulation methods require both variables to be linearly related and to exhibit a homoscedastic behaviour (correlation of both variables is constant over the range of values considered), BSS can handle virtually any relationship between the primary and the secondary variables and does not require explicit knowledge of the corresponding cross-variogram. An inherent limitation of BSS is that it can only account for a single collocated secondary variable, thus, as collocated cosimulation, assumes MM2. The method was initially applied to the simulation of lithoclasses based on seismic impedance measurements (Doyen \& Boer 1996). Later, Dubreuil-Boisclair et al. (2011) used BSS to estimate the local hydraulic conductivity distribution based on cross-hole ground-penetrating radar tomograms and borehole-based hydraulic conductivity measurements from slug tests and flowmeter measurements. Ruggeri et al. $(2013,2014)$ then adapted the method to allow for the simulation of larger-scale heterogeneous hydraulic conductivity fields guided by information from spatially extensive, but poorly resolved tomographic images of surface-based geoelectric measurements. To our knowledge, this is the first approach of this kind that allowed for hydrogeophysical data integration to be extended from the predominantly local to the subregional scale.

While BSS is recognized as a powerful and flexible geostatistical tool (e.g. Ezzedine et al. 1999; Chen et al. 2001; Doligez et al. 2015), it also presents significant and, as of yet, unresolved challenges. In particular, the simultaneous reproduction of (1) the variance and the fine-scale structure and (2) the relationship between the primary and the secondary variables has proven to be elusive. In the context of the hydrogeophysical applications mentioned above, the difficulties are exacerbated by the smooth distribution of the secondary variable due to the regularization typically used by geophysical inversion procedures. To overcome this problem, Ruggeri et al. (2013, 2014) added several algorithmically complex and computationally expensive steps to BSS in order to ensure an adequate reproduction of the underlying statistics. The first step was to downscale the smooth electrical conductivity structure inferred through surface-based electrical resistivity tomography (ERT) to the desired resolution. A second step consisted of gradual deformation (Hu et al. 2001) to make sure that the downscaled electrical conductivity structure matched the original coarse-scale measurements while simultaneously retaining the correct fine-scale characteristics. In a third step, classical BSS was used to simulate the hydraulic conductivity distribution based on the downscaled electrical conductivity field and the original hydraulic conductivity data.

The primary reason for the inability of BSS to directly combine the information of the primary variable with that of the secondary variable lies in the inherent assumption of conditional independence between the neighbours of the simulated cell and the collocated secondary variable (Doyen et al. 1996). The objective of this study is to address and overcome this limitation by accounting for this interdependence, thus enabling BSS-based hydrogeophysical data integration in one single step. To this end, the Bayesian aggregation of the primary and secondary information is generalized in the context of a probability aggregation framework (e.g. Genest \& Zidek 1986) known as log-linear pooling, which relaxes the assumption of conditional independence by adding exponential weights to the probabilities conditional to the primary and secondary information (e.g. Allard et al. 2012). Similar approaches have already been used for various applications in geosciences, such as the interpolation of satellite images (e.g. Mariethoz et al. 2009b), the reconstruction of 3-D volumes based on 2-D sections (e.g. Comunian et al. 2012) and the inclusion of auxiliary information in multipoint geostatistical simulations (e.g. Hoffimann et al. 2017).

\section{METHODOLOGICAL BACKGROUND}

\subsection{Classical BSS}

The input data for BSS typically consist of highly resolved, yet spatially sparse, measurements of the primary variable $X$, also referred to as hard data, and spatially exhaustive, yet poorly resolved, estimates of the secondary variable $Z$. No a priori relationship between the primary and the secondary variables is assumed and, therefore, any suitable joint distribution $p(X, Z)$ can be used. In previous works, this joint distribution was inferred from the hard data and corresponding collocated values of the secondary variable (Dubreuil-Boisclair et al. 2011; Ruggeri et al. 2013; Ruggeri et al. 2014).

The procedure followed by BSS for each simulated cell is the following (Fig. 1):

(1) An unpopulated cell $X_{i}$ is randomly selected on the grid of the primary variable. 


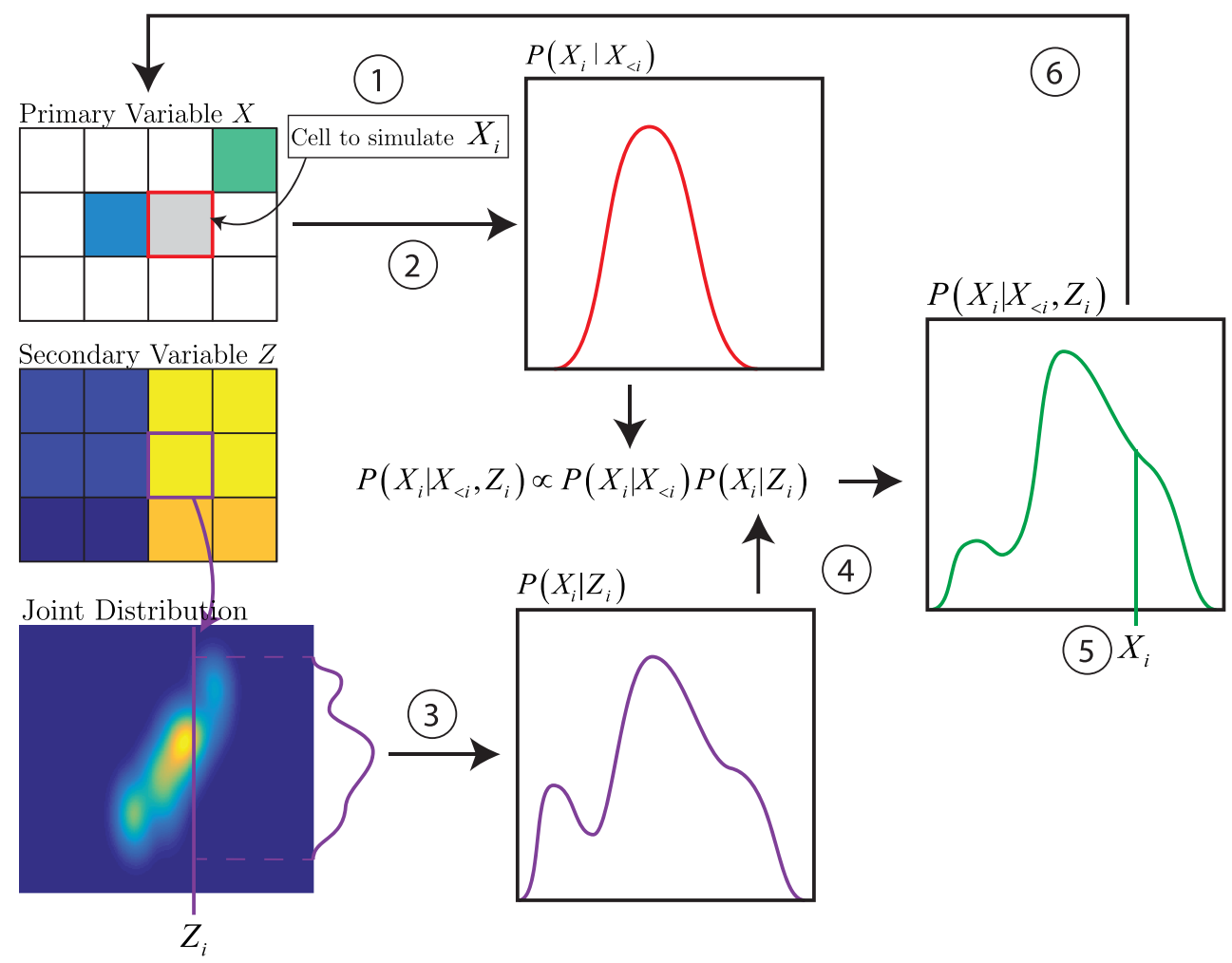

Figure 1. Schematic illustration of the classical BSS approach: (1) Selection of the unknown cell to simulate $X_{i}$, (2) kriging estimate of the measured and previously simulated values of the primary variable $P\left(X_{i} \mid X_{<i}\right)$, (3) estimation of the marginal distribution from the joint probability distribution of the primary $X_{i}$ and secondary $Z_{i}$ variable, (4) determination of the posterior distribution, (5) random sampling of the posterior distribution and (6) assignment of the sampled value to the selected cell.

(2) Using kriging, the conditional distribution $P\left(X_{i} \mid X_{<i}\right)$ is computed based on the neighbouring cells, denoted as $X_{<i}$, which may include hard data and previously simulated cells. If $X$ is not Gaussian, a normal-score transform is applied to the neighbouring values prior to kriging and then the conditional distribution $P\left(X_{i} \mid X_{<i}\right)$ is back-transformed to the non-Gaussian space.

(3) The conditional distribution $P\left(X_{i} \mid Z_{i}\right)$ is extracted from the joint distribution $p(X, Z)$ based on the known collocated secondary variable $Z_{i}$, which essentially assumes that $P\left(X_{i} \mid Z_{i}\right) \propto P\left(Z_{i} \mid X_{i}\right)$. The two distributions $P\left(X_{i} \mid X_{<i}\right)$ and $P\left(Z_{i} \mid X_{i}\right)$ are then combined through Bayesian updating,

$P\left(X_{i} \mid X_{<i}, Z_{i}\right) \propto P\left(X_{i} \mid X_{<i}\right) P\left(Z_{i} \mid X_{i}\right)$.

(4) A value for $X_{i}$ is sampled from the resulting distribution.

(5) The grid is updated with the new value.

\subsection{Conditional independence in Bayesian updating}

In the following, the inherent assumption of conditional independence made in BSS is highlighted and its consequences are discussed. It can be shown (Appendix A) that the exact decomposition of the conditional probability $P\left(X_{i} \mid X_{<i}, Z_{i}\right)$ is

$$
P\left(X_{i} \mid X_{<i}, Z_{i}\right) \propto P\left(X_{i} \mid X_{<i}\right) P\left(Z_{i} \mid X_{i}, X_{<i}\right) .
$$

However, the Bayesian updating used by BSS in eq. (1) assumes that the secondary variable $Z_{i}$ is independent of the previously simulated points of the primary variable $X_{<i}$, conditional to the simulated point $X_{i}$ (Doyen et al. 1996), which can be written as

$P\left(Z_{i} \mid X_{i}, X_{<i}\right)=P\left(Z_{i} \mid X_{i}\right)$.

This assumption, referred to as conditional independence, implies that all the information related to $Z_{i}$ that is included in $X_{<i}$ is also included in $X_{i}$. In other words, $X_{i}$ screens the influence of $X_{<i}$ related to $Z_{i}$. To assess the consequences of this assumption, we consider the special case of a joint multi-Gaussian random field $X$ and $Z$. Doyen et al. (1996) showed the equivalence of Bayesian updating with strictly collocated cokriging, for which a type 1 Markov model (MM1) is assumed (Xu et al. 1992; Chilès \& Delfiner 1999, p. 311; Journel 1999). As noted in Doyen et al. (1996), the main advantage is to decouple the respective influences of the primary and secondary variables, thus avoiding the need to determine a cross-covariance. While MM1 is appropriate for scenarios with extensive knowledge of the secondary variable, it is only applicable if the measurements associated with the primary and secondary variables have the same support volume (Journel 1999). Thus, 


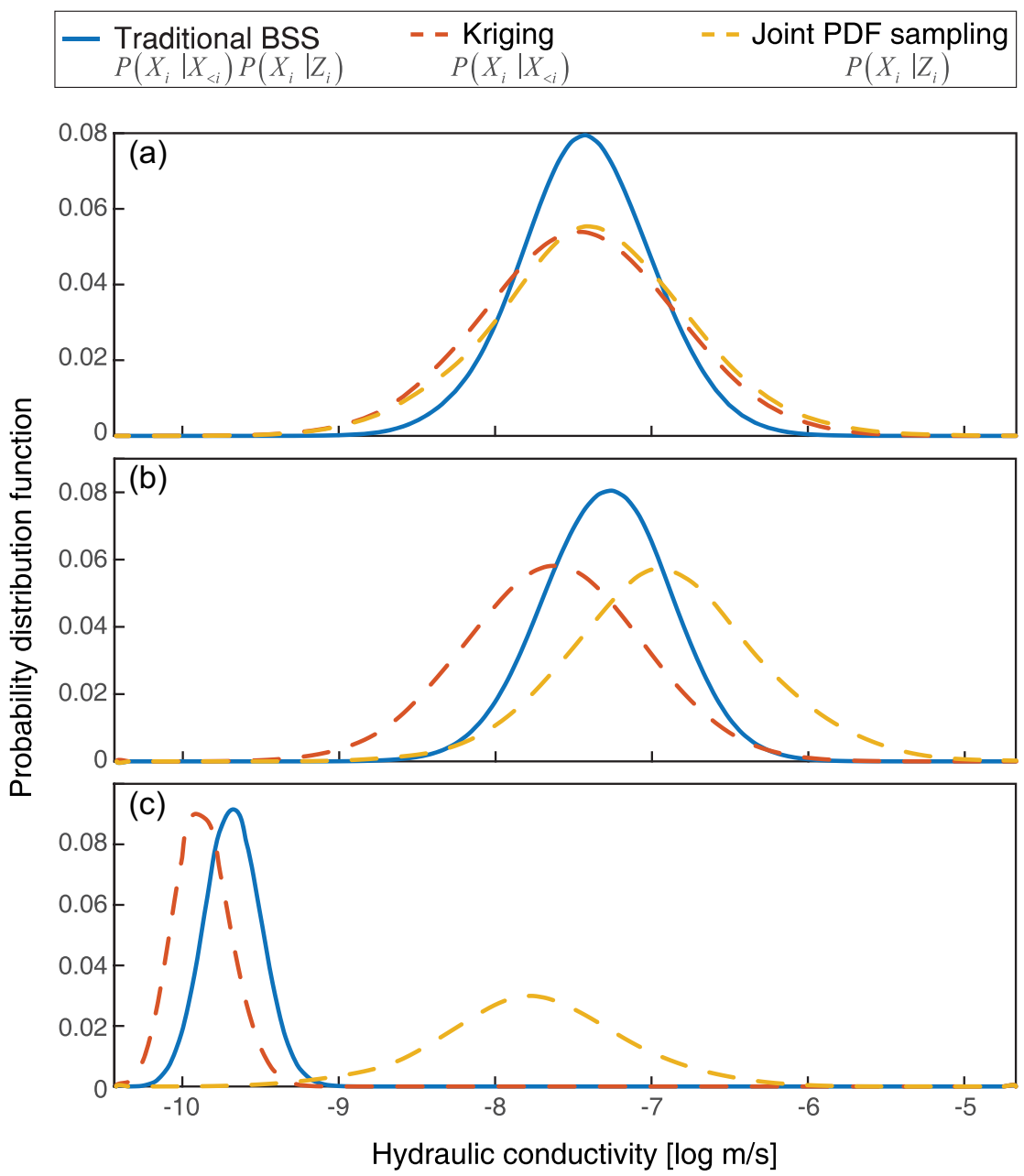

Figure 2. Illustration of the effects of assuming conditional independence in classical BSS for (a) a cell located at the bottom of the domain simulated at the beginning of the simulation, (b) a cell located near the surface and also simulated at the beginning and (c) a cell simulated towards the end.

in a typical hydrogeophysical scenario, the assumption of MM1 is hardly justifiable because the spatial distribution of the secondary variable tends to be significantly smoother than that of the primary variable. Moreover, the sequential nature of BSS makes it especially sensitive to this assumption, as any bias introduced is amplified in the course of the simulation.

We focus on the assumption of conditional independence within MM1. However, BSS also implicitly ignores the values of the secondary variable at non-collocated cells when using the left-hand side of eq. (1). In the Gaussian case, this simplification is equivalent to reducing cokriging to strictly collocated cokriging. The validity of collocated (MM2) and strictly collocated cokriging is extensively discussed by Rivoirard (2001; 2004) and Journel (1999). In the BSS framework, there is no equivalence to non-strictly collocated cokriging as the joint probability distribution function (pdf) is only built using collocated primary and secondary variables.

The first scenario considered corresponds to a cell simulated at the beginning of the simulation path, which, therefore, is poorly informed by its neighbours (Fig. 2a). Moreover, this specific cell is located at the bottom of the domain, where the secondary variable is not very informative. Consequently, both $P\left(X_{i} \mid Z_{i}\right)$ and $P\left(X_{i} \mid X_{<i}\right)$ produce estimates that are close to the marginal distribution. However, classical BSS treats them as being independent and generates a distribution with an inaccurate variance that is smaller than the marginal. The second scenario also corresponds to a cell simulated at the beginning of the process (Fig. 2b). However, this cell is located near the top of the domain where the secondary variable is informative and is located far from any hard data. In this case, kriging is poorly constrained, which results in a distribution close to the marginal. In such a situation, classical BSS fails to neglect the kriging estimate. The cell corresponding to the third scenario is among the last ones to be simulated (Fig. 2c). Correspondingly, kriging provides a very well constrained estimation since the neighbours are close to the simulated cell. In contrast, the secondary variable provides an estimation similar to the marginal distribution because the location of the cell near the bottom of the domain makes the secondary variable poorly informative. In this case, classical BSS is not able to determine that the secondary information has already been included into the simulation of the neighbouring cells and, hence, should not be included again.

The three examples highlight essential limitations of BSS in its classical form and illustrate the need for a method, which is capable of weighing the relative importance of both sources of information. The latter can be achieved by resorting to approaches known as probability aggregation (e.g. Allard et al. 2012), which we explore in the following. 


\subsection{BSS with log-linear pooling}

Probability aggregation provides a general framework to combine estimations of an event $A$, where each estimation is based on different data $\left\{D_{1}, \ldots, D_{n}\right\}$ with unknown dependences. A pooling operator $F$ is defined to approximate the conditional distribution $P\left(A \mid D_{1}, \ldots, D_{n}\right)$ based on the individual conditional probabilities $P\left(A \mid D_{i}\right)$ :

$P\left(A, D_{1}, \ldots, D_{n}\right) \approx F\left(P(A), P\left(A \mid D_{1}\right), \ldots, P\left(A \mid D_{n}\right)\right)$.

Allard et al. (2012) described several types of pooling operators $F$, from which the log-linear operator is one of the most generic and most popular product-based ones:

$P\left(A, D_{1}, \ldots, D_{n}\right) \propto P(A)^{1-\sum_{i} w_{i}} \prod_{i=1}^{n} P\left(A \mid D_{i}\right)^{w_{i}}$.

Allard et al. (2012) showed that the log-linear pooling decomposition is exact, that is, it accounts for all dependences, when the weights $w_{i}$ are computed as

$w_{i}=\frac{\ln P\left(D_{i} \mid A, D_{<i}\right)}{\ln P\left(D_{i} \mid A\right)}$.

Yet, as the numerator of eq. (6) is usually unknown, the weights have to be approximated. If $w_{i}=1$, the numerator and denominator of eq. (6) become equal, such that the data $\left\{D_{1}, \ldots, D_{n}\right\}$ are assumed to be independent conditional to $A$. This kind of aggregator corresponds to the so-called conjunction of probability (Tarantola 2005). If $\sum_{i} w_{i}=1$, the prior distribution $P(A)$ vanishes in eq. (5) and the pooling preserves unanimity. Unanimity implies that the pooling aggregation results in the same probability, if all individual conditional probabilities are equal.

In the context of BSS, log-linear pooling can be used to combine the information provided by the kriging $P\left(X_{i} \mid X_{<i}\right)$ and by the joint probability $P\left(X_{i} \mid Z_{i}\right)$ such that the assumption of independence is relaxed through the use of the weights $w_{X}$ and $w_{Z}$. Eq. (5) can therefore be rewritten as

$P\left(X_{i} \mid Z_{i}, X_{<i}\right) \propto P\left(X_{i}\right)^{1-w_{X}-w_{Z}} \cdot P\left(X_{i} \mid Z_{i}\right)^{w_{Z}} \cdot P\left(X_{i} \mid X_{<i}\right)^{w_{X}}$.

In this paper, $P\left(X_{i}\right)$ is referred to as the prior, while $P\left(X_{i} \mid X_{<i}\right)$, considered the prior in classical BSS, is referred to as a conditional probability because it is based on information from the neighbours. The prior can either be assumed to be unknown and chosen as a uniform distribution, thus having no influence, or it can be assumed to correspond to the marginal distribution.

\subsection{Weighting schemes and objective functions}

The proposed formulation of BSS with log-linear pooling in eq. (7) allows us to mitigate the effects related to the dependences between the information of the primary and secondary variables. This approach does, however, require an appropriate choice of the weights $w_{X}$ and $w_{Z}$, for which there are, as of yet, no rules or guidelines. We therefore explore four canonical strategies for choosing and/or estimating suitable weights, which we refer to as (1) BSS-0.5 and BSS-1, (2) Cst-BSS, (3) Step-BSS and (4) Multi-step-BSS.

The simplest weighting approach comprises BSS-0.5 and BSS-1, for which the weights are chosen as $w_{X}=w_{Z}=0.5$ and $w_{X}=w_{Z}=1$, respectively. In the Cst-BSS approach, the optimal values for $w_{X}$ and $w_{Z}$ are inferred through a calibration procedure. For Step-BSS, the weights are no longer constant during the entire simulation process, but, at a given point, change abruptly from $w_{X}=0$ and $w_{Z}=1$ to $w_{X}=1$ and $w_{Z}=0$. We seek to constrain the optimal timing of this switch, as quantified by the fraction of the grid that has been simulated to this point. Finally, Multi-step BSS changes the values of the weights multiple times during the simulation process based on an exhaustive Monte-Carlo-type search (Appendix B). In the following, we test the viability of these weighting schemes based on a synthetic case study and discuss the corresponding results.

To compare and calibrate these weighting schemes, they are evaluated by their ability to reproduce (1) the relation between primary and secondary variables and (2) the underlying geostatistical model. These criteria are assessed through two specific objective functions. The first criterion is evaluated by the mismatch between the joint pdf of the realizations $p_{x^{(i)}}(X, Z)$ and the joint reference pdf of the underlying model $p(X, Z)$, which is achieved by computing the root-mean-square error of the discrete joint distributions

$\mathrm{OF}_{Z}=\frac{1}{n_{u} n_{v}} \sqrt{\sum_{u=1}^{n_{u}} \sum_{v=1}^{n_{v}}\left(\frac{1}{n} \sum_{i=1}^{m} p_{x^{(i)}}\left(X=x_{u}, Z=z_{v}\right)-p\left(X=x_{u}, Z=z_{v}\right)\right)^{2}}$.

The second criterion is assessed by comparing the empirical variograms of the realizations $\gamma_{x^{(i)}}$ with the model variogram $\gamma_{X}$. The corresponding objective function is quantified by the root-mean-square-type error at each discrete vertical and horizontal lag distance $\mathbf{h}_{j}$ for lags up to two times the variogram range:

$\mathrm{OF}_{X}=\left\|\frac{1}{\mathbf{K}} \sqrt{\sum_{j=1}^{n_{j}} k\left(\mathbf{h}_{j}\right)\left(\frac{1}{n_{i}} \sum_{i=1}^{n_{i}}\left(\gamma_{x^{(i)}}\left(\mathbf{h}_{j}\right)-\gamma_{X}\left(\mathbf{h}_{j}\right)\right)\right)^{2}}\right\|_{1}$ 


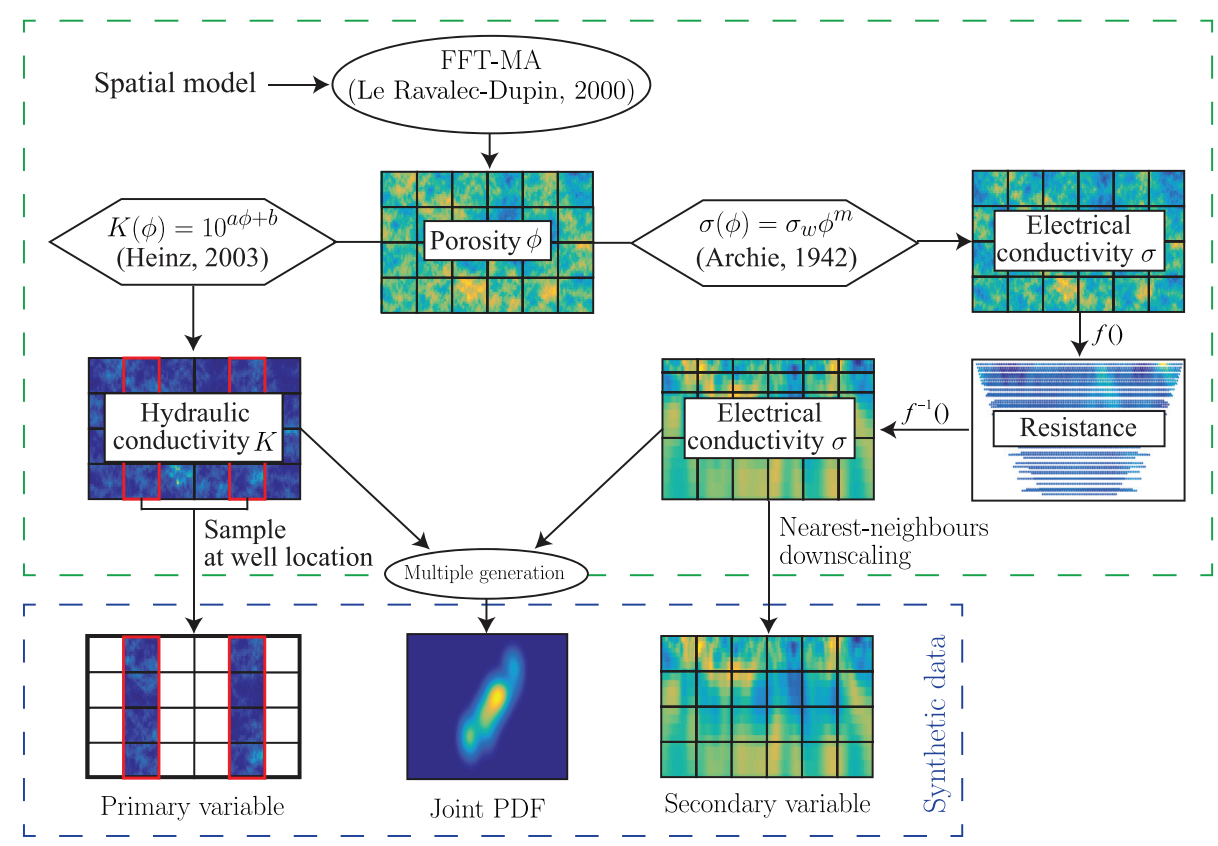

Figure 3. Illustration of the procedure used to generate the synthetic hydrogeophysical database considered in this study. A heterogeneous porosity field is generated and transformed into corresponding hydraulic and electrical conductivity fields. The large-scale electrical conductivity structure is inferred through surface-based geoelectric measurements and their subsequent tomographic inversion. The fine-scale hydraulic conductivity structure is sampled along isolated boreholes.

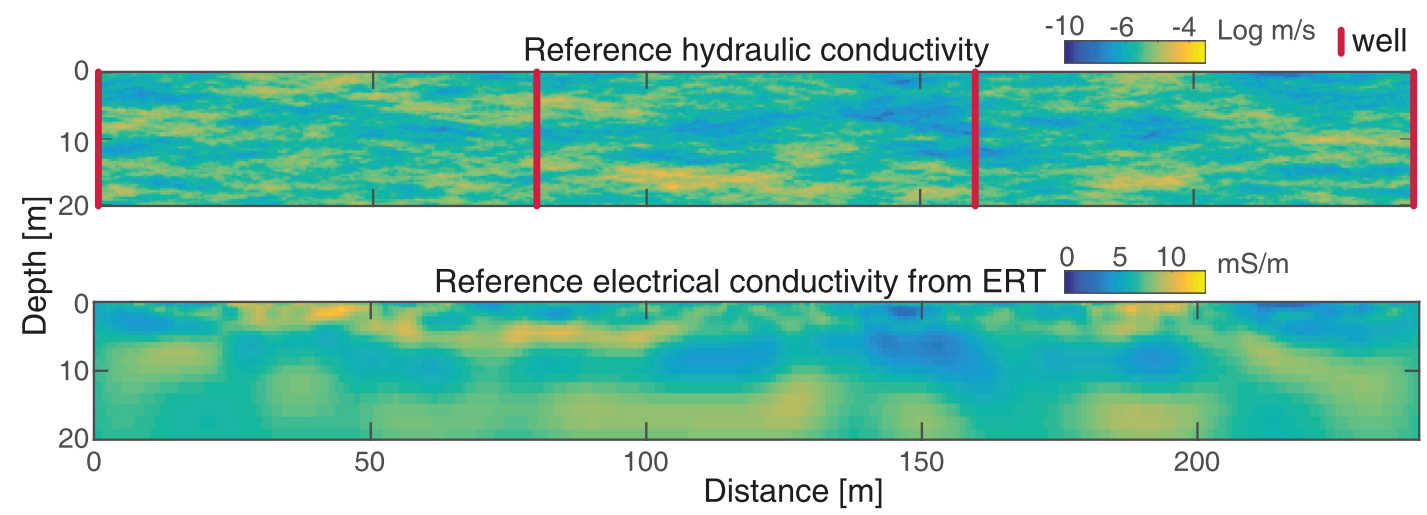

Figure 4. Top: reference distribution of hydraulic conductivity; bottom: low-resolution electrical conductivity structure inferred from ERT-type inversion of surface-based geoelectric measurements. The red vertical lines denote boreholes, along which the hydraulic conductivity is assumed to be known.

where $\mathbf{K}=\sum_{j=1}^{n_{j}} k\left(\mathbf{h}_{j}\right)$ and $k\left(\mathbf{h}_{j}\right)=1-\gamma_{X}\left(\mathbf{h}_{j}\right)$ weigh the misfit at each lag distance based on the corresponding value of the model variogram. This criterion favours the reproduction of the model variogram at shorter lags and, hence, is particularly sensitive to the small-scale heterogeneity. The two objective functions $\mathrm{OF}_{X}$ and $\mathrm{OF}_{Z}$ do not depend on the true field so that they can be readily computed in real-world scenarios where the corresponding values are unknown. Because of ergodic fluctuations in the simulations, the objectives functions need to be computed for multiple realizations.

\section{SYNTHETIC HYDROGEOPHYSICAL CASE STUDY}

To test the proposed method and the associated weighting schemes, we consider a synthetic hydrogeophysical case study, which is largely identical to that used by Ruggeri et al. (2013) for their multistep approach using classical BSS. The primary variable corresponds to the hydraulic conductivity and the secondary variable to the electrical conductivity field from an ERT-type deterministic inversion of surface-based geoelectric data. The procedure used to generate this synthetic hydrogeophysical database is outlined below and illustrated in Figs 3 and 4 .

1. A heterogeneous Gaussian porosity field $\phi$ is generated with the fast Fourier transform moving average (FFT-MA) method (Le RavalecDupin et al. 2000) on a $240 \times 20 \mathrm{~m}$ domain consisting of $513 \times 65$ cells. This porosity distribution is characterized by a mean value of 0.27 , a standard deviation of 0.05 and a 2-D exponential variogram having horizontal and vertical ranges of $27 \mathrm{~m}$ and $2.7 \mathrm{~m}$, respectively. 
2. From this porosity field, both the hydraulic and the electrical conductivity fields are generated based on petrophysical relationships.

3. The electrical conductivity field is computed using Archie's law (Archie 1942) for full saturation $\sigma(\phi)=\sigma_{w} \phi^{m}$ with an electrical conductivity of the pore water $\sigma_{w}$ of $43 \mathrm{mS} \mathrm{m}^{-1}$ and a cementation exponent $m$ of 1.4.

4. The fine-scale hydraulic conductivity structure is obtained through an empirical power-law relationship of the form $K(\phi)=10^{a \phi+b}$ (Heinz et al. 2003) with $a=6.66$ and $b=4.97$.

5. The synthetic hydraulic and electrical conductivity fields are then subjected to typical hydraulic and geophysical measurement approaches, respectively.

6. The hydraulic conductivity is sampled along four equally spaced boreholes and constitutes the high-resolution hard data, which could be obtained, for example, through flowmeter and/or slug test measurements (e.g. Dubreuil-Boisclair et al. 2011). The primary variable is taken as the normal score transform of the logarithm of the hydraulic conductivity. The corresponding back-transformation is performed at the end of the simulation.

7. The secondary variable is generated from the electrical conductivity field in two steps. First, a forward surface-based geoelectric survey is simulated on the 'true' electrical field leading to a series of resistance. Then, the resulting resistance data are inverted using a least-squares-type deterministic algorithm producing a heavily smoothed estimate of the electrical conductivity distribution, which constitutes the secondary variable. Both steps are performed using the software R2 (Binley \& Kemna 2005). For computational reasons, it is common to perform the inversion on a coarse grid, which has essentially no effect on the final tomographic image, because of the smoothing associated with the deterministic regularization of the inversion process. However, in order to adequately reflect the change of resolution with depth, which is inherent to surface-based ERT images, the inversion grid has a logarithmic vertical spacing. Finally, to use this ERT image in BSS, a nearest-neighbour downscaling to the grid size of the primary variable is performed.

8. The joint distribution of the primary and secondary variables is traditionally inferred using the collocated samples at the borehole locations. However, to be representative of the area of interest, numerous samples are needed. In practice, it is therefore common to use data from related and/or analogue sites. We mimic this approach by repeating the procedure to generate several unconditional simulations of both the primary and the secondary variables (steps 1-3 above). All these fields are then used to build the joint distribution. The proposed approach allows quantifying the performance of our approach without contamination from other sources of uncertainty, in particular, a biased joint pdf resulting from a suboptimal number of samples.

The kriging part of BSS uses a neighbourhood search strategy consisting of a two-part search with a spiral search of 40 cells for the previously simulated cells and a superblock search of 20 cells for the hard data (Deutsch \& Journel 1992). A parallelized constant multigrid simulation path is used to optimize the computational efficiency while simultaneously minimizing the simulation biases (Nussbaumer et al. 2018).

\section{RESULTS}

We applied the four different weighting schemes outlined above to the considered synthetic hydrogeophysical data example (Fig. 4). The key results, which are summarized in terms of the associated values of the horizontal and vertical objective functions $\mathrm{OF}_{X}$ and $\mathrm{OF}_{Z}$ (Fig. 5), will be discussed in the following. The true field has non-zero values for $\mathrm{OF}_{X}$ because of ergodic fluctuations of the variogram (e.g. Emery 2004) and because of the sampling bias of the joint pdf when using a single realization, respectively. Similarly, the small differences between SGS, Cst-BSS for $w_{X}=1, w_{Z}=0$, and Step-BSS for $T=0$ are also due to the ergodic fluctuation.

\subsection{BSS- -0.5 and BSS-1}

We compare two basic aggregating schemes (BSS-0.5 and BSS-1) to classical BSS and related stochastic simulation techniques to explore the potential advantages of using log-linear pooling (Table 1). Classical BSS can be obtained from eq. (7) by setting $w_{Z}=w_{X}=1$ and assuming a uniform, that is, an unknown prior. For $w_{X}=1$ and $w_{Z}=0$, only the kriging information is used such that the procedure becomes identical to SGS. Conversely, using $w_{X}=0$ and $w_{Z}=1$, results in so-called white cosimulation, where only the information of the secondary variable is used. Additionally, we consider two simple weighting schemes, referred to as BSS-0.5 and BSS-1, which honour the condition of log-linear pooling. Neither of these schemes applies any preference to $P\left(X_{i} \mid X_{<i}\right)$ or $P\left(X_{i} \mid Z_{i}\right)$. The property of unanimity is preserved for BSS-0.5, which uses $w_{Z}=w_{X}=0.5$ so that the prior is not included as $1-w_{X}-w_{z}=0$. Conversely, BSS-1 sets the weights to $w_{Z}=w_{X}=1$ and uses the marginal distribution of the primary variable as the prior with a weight of $1-w_{X}-w_{z}=-1$.

To illustrate the implications of using these four weightings schemes, we illustrate their respective aggregating equation $P\left(X_{i} \mid Z_{i}, X_{<i}\right)$ when applied to the same three examples of Section 2.2 (Figs 2 and 6). By definition, SGS and white cosimulation result in the same distributions as the kriging estimate $P\left(X_{i} \mid X_{<i}\right)$ and the joint distribution $P\left(X_{i} \mid Z_{i}\right)$, respectively.

In the first scenario (Fig. 6a), the aggregation used in classical BSS leads to a reinforcement of the central value as both sources of information are considered to be independent. The other aggregation methods mitigate this effect and result in estimates with larger variances. For instance, because of its unanimity property, the estimation provided by BSS-0.5 is almost identical to both $P\left(X_{i} \mid X_{<i}\right)$ and $P\left(X_{i} \mid Z_{i}\right)$.

In the second scenario (Fig. 6b), $P\left(X_{i} \mid X_{<i}\right)$ and $P\left(X_{i} \mid Z_{i}\right)$ have the same variance, but a different expected value. If $P\left(X_{i} \mid X_{<i}\right)$ and $P\left(X_{i} \mid Z_{i}\right)$ provide independent information, classical BSS succeeds in finding the overlapping estimate. However, when there is 


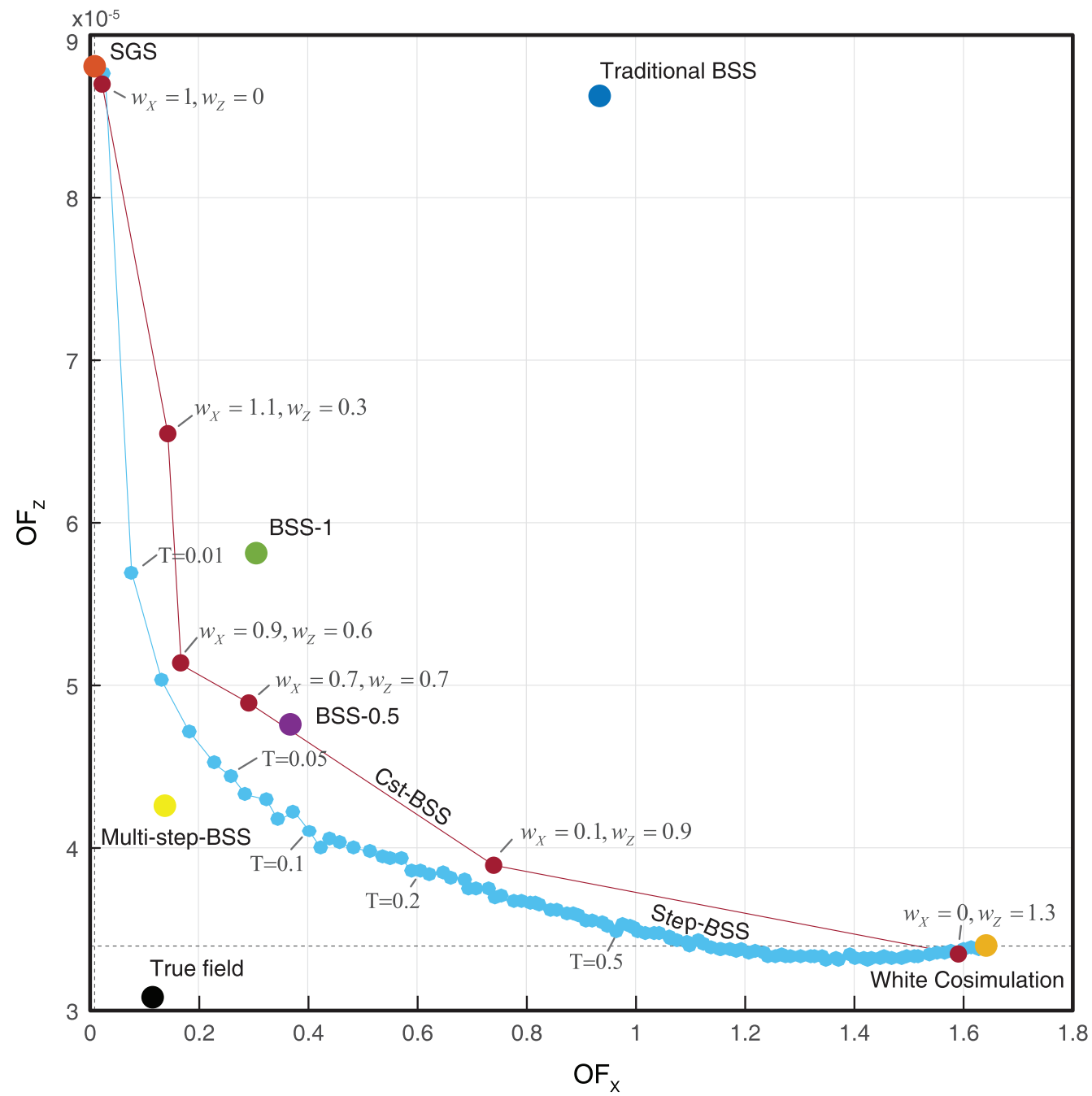

Figure 5. Performance of the different weighting schemes considered in this study with regard to the reproduction of the variogram and the joint distribution as quantified by the objective functions $\mathrm{OF}_{X}$ and $\mathrm{OF}_{Z}$, respectively. Cst-BSS shows the optimal weights associated with the Pareto front. Step-BSS displays all step positions tested, where $T$ denotes the fraction of the grid that has been simulated when the weights are switched. Multi-step-BSS shows the global optimum inferred when allowing flexible adaptations of the weights in the course of the simulation process. The two dotted lines illustrate the normalization factors computed using eq. (10).

Table 1. Comparison of some key characteristics of basic aggregation schemes considered in this study.

\begin{tabular}{lccc}
\hline & Aggregating equation for $P\left(X_{i} \mid Z_{i}, X_{<i}\right)$ & $w_{X}$ & $w_{Z}$ \\
\hline Classical BSS & $P\left(X_{i} \mid Z_{i}\right) \cdot P\left(X_{i} \mid X_{<i}\right)$ & 1 & 1 \\
SGS & $P\left(X_{i} \mid X_{<i}\right)$ & 1 & 0 \\
White cosimulation & $P\left(X_{i} \mid Z_{i}\right)$ & 0 & 1 \\
BSS-0.5 & $\sqrt{P\left(X_{i} \mid Z_{i}\right) \cdot P\left(X_{i} \mid X_{<i}\right)}$ & 0.5 & 0.5 \\
BSS-1 & $\frac{P\left(X_{i} \mid Z_{i}\right) \cdot P\left(X_{i} \mid X_{<i}\right)}{P\left(X_{i}\right)}$ & 1 & 1 \\
\hline
\end{tabular}

interdependence, it overestimates the agreement between $P\left(X_{i} \mid X_{<i}\right)$ and $P\left(X_{i} \mid Z_{i}\right)$, hence, supresses all values present in only one of the distributions. As a consequence, the estimate has a small variance and tends to the marginal distribution $P_{0}(X)$. BSS- 1 corrects for this by including the marginal distribution with a weight of -1 and produces an estimate with a larger variance.

The third scenario (Fig. 6c) depicts a situation where $P\left(X_{i} \mid X_{<i}\right)$ and $P\left(X_{i} \mid Z_{i}\right)$ provide fundamentally different estimate, both in terms of the mean and the variance. BSS- 0.5 and BSS-1 give estimates that are similar to $P\left(X_{i} \mid X_{<i}\right)$ because they favour estimations with smaller variance. On the other hand, classical BSS favours overlaps between estimations, which results in a distribution with low variance, and thus explains why it tends to underrepresent extreme values in the realizations.

The differences between these approaches are illustrated with one realization of each weightings scheme (Fig. 7), and further assessed by quantifying the reproduction of the joint pdf (Fig. 8) and the variogram (Fig. 9) using 480 realizations computed for each weighting scheme. 


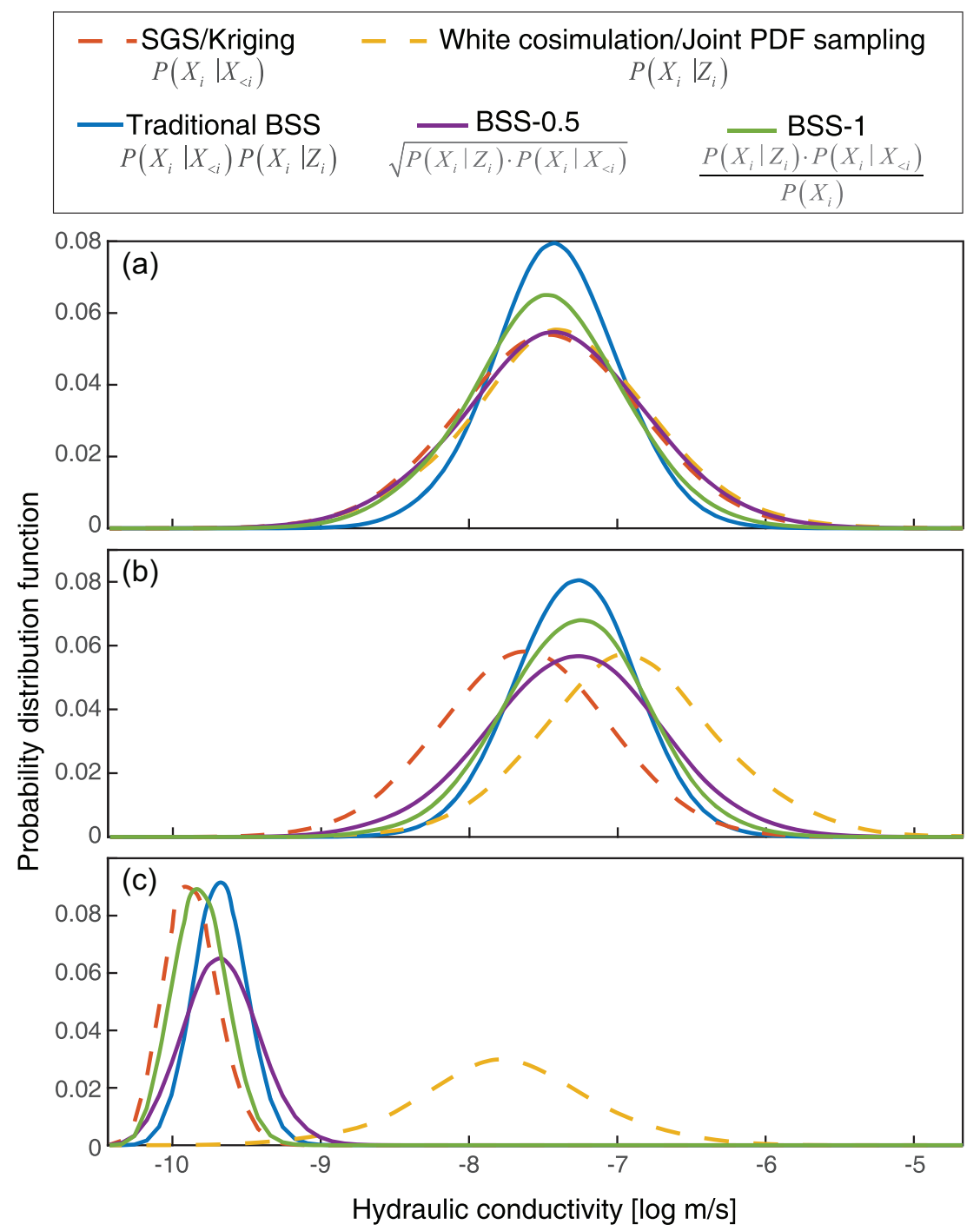

Figure 6. Probability distributions for the weighting schemes given in Table 1 for three scenarios related to $P\left(X_{i} \mid X_{<i}\right)$ and $P\left(X_{i} \mid Z_{i}\right)$ : (a) equal mean and variance, (b) equal variance but different mean and (c) different mean and different variance.

The simulations generated with SGS have a similar fine-scale structure as the reference field and, hence, the corresponding empirical variogram follows closely that of the reference (Fig. 9). Yet, as the values of the field distant from boreholes are unconstrained, the joint distribution is not well reproduced (Fig. 8). In contrast, white cosimulation offers the best reproduction of the large-scale features (Fig. 7), but ignores the fine-scale structure, which results in noisy realizations that fail to reproduce the target variogram at small lags (Fig. 9). Classical BSS combines both sources of information and, therefore, reproduces the joint distribution better than SGS (Fig. 8) and the variogram better than white cosimulation (Fig. 9). However, as it assumes conditional independence of the primary and secondary variable, it tends to reduce the variance (Figs 7 and 9). Both log-linear pooling weighting schemes (BSS-0.5 and BSS-1) improve the quality of realizations with respect to the reproduction of the joint distribution (Fig. 8) and the variogram (Fig. 9). In general, realizations of BSS-0.5 result in a slightly better joint distribution, while BSS-1 provides a slightly better reproduction of the target variogram, primarily at small lag distances. The comparison between weighting schemes is summarized in terms of $\mathrm{OF}_{X}$ and $\mathrm{OF}_{Z}$ (Fig. 5). SGS provides the best results with respect to OF ${ }_{Z}$, but at the expense of a poor score in terms of $\mathrm{OF}_{X}$. The opposite situation occurs for white cosimulation. This comparison illustrates the improvement provided by BSS-1 and BSS- 0.5 compared to classical BSS with respect to both $\mathrm{OF}_{X}$ and $\mathrm{OF}_{Z}$.

Finally, the assessment of the weightings schemes based on $\mathrm{OF}_{X}$ and $\mathrm{OF}_{Z}$ needs to be discussed. When assessing a variogram, the aggregation of the errors at each lag distance can create a bias in favour of short (i.e. fine-scale structure) or long lag distances (i.e. variance). The formulation of $\mathrm{OF}_{X}$ in eq. (9) is designed to strike a balance by weighing the errors of each lag according to the value of the variogram at this lag. A possible alternative would be to normalize the realizations after the simulation and before computing $\mathrm{OF}_{X}$. As illustrated by the sill of the variogram of classical BSS (Fig. 7), the corresponding $\mathrm{OF}_{X}$ value would decrease. However, this correction is difficult to apply because it would alter the value of hard data as well as affecting the reproduction of $\mathrm{OF}_{Z}$. Similarly, the root-mean-square approach used to measure the reproduction of the joint pdf in eq. (10) tends to emphasize individual larger errors compared to multiple smaller errors. As a 


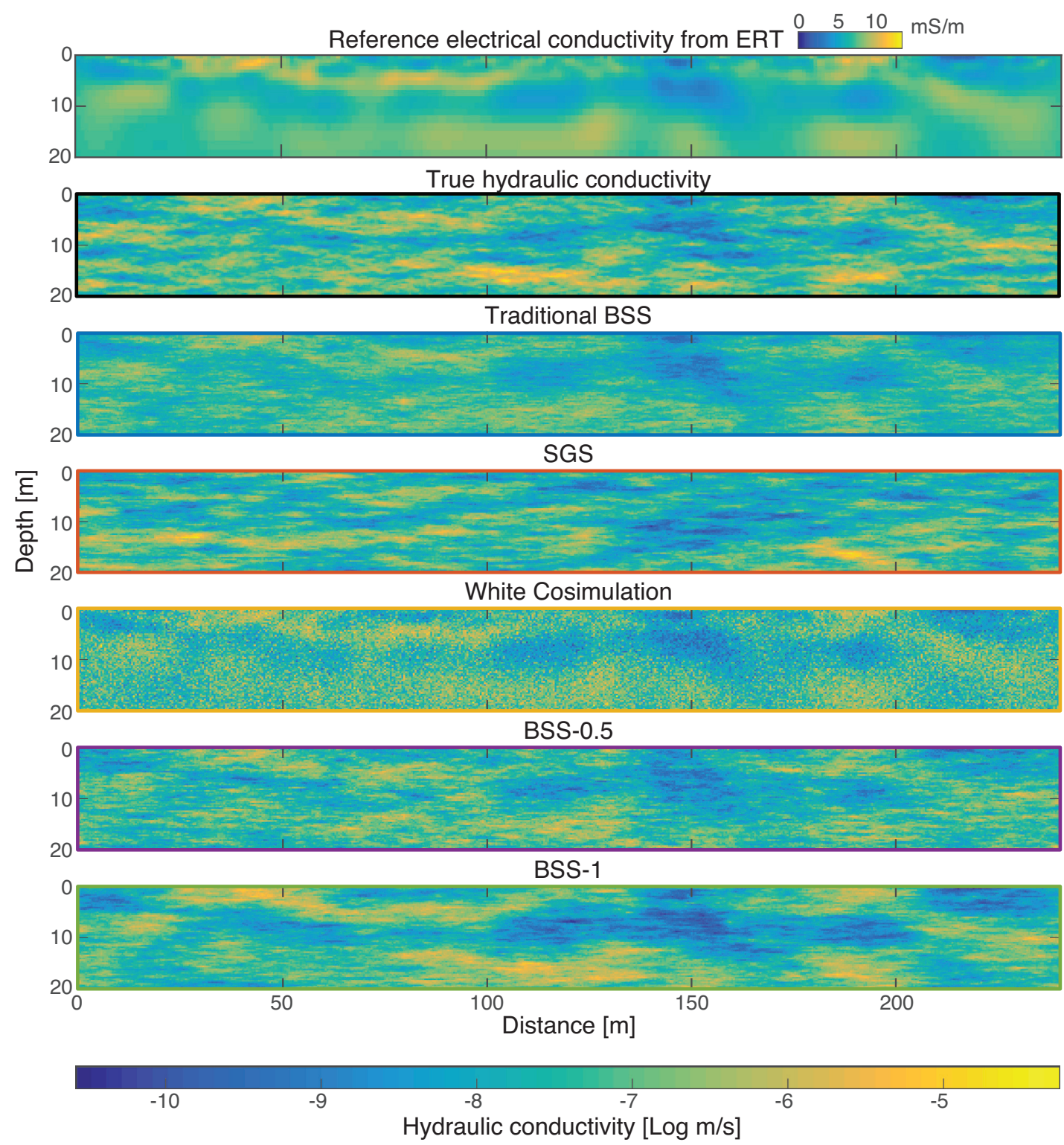

Figure 7. Comparison of the secondary variable (first from top) and the true reference field of the primary variable (second from top) to individual realizations for each of the scenarios described in Table 1. The realization resulting from classical BSS is comparatively smooth. The realization using SGS produces the correct fine-scale characteristics. The realization using white-cosimulation matches well the secondary variable. The realizations based on BSS-0.5 and BSS-1 strikes a balance between SGS and white cosimulation.

result, while classical BSS accurately reproduces the shape of the joint pdf (Fig. 8), its $\mathrm{OF}_{Z}$ value is relatively high due to the large magnitude of its errors.

\subsection{Cst-BSS}

In the Cst-BSS approach, the optimal values of the weights $w_{X}$ and $w_{Z}$ are inferred through an exhaustive grid search. To the end, the objective functions $\mathrm{OF}_{\mathrm{X}}$ and $\mathrm{OF}_{\mathrm{Z}}$ are computed for simulations with weights ranging from 0 to 2 with an increment of 0.1 . For each test, 96 realizations are used. Proceeding in this manner then allows to infer the Pareto front identifying the ensemble of weights resulting in the minimum of any linear combination of both objective functions (Fig. 10). The objective functions are normalized based on the two end-member scenarios SGS $\left(w_{X}=1, w_{Z}=0\right)$ and white cosimulation $\left(w_{X}=0, w_{Z}=1\right)$. The Pareto front is identified by minimizing the linear combinations of the normalized objective functions scaled by a parameter $t$ varying from 0 to 1 :

$$
\min _{w_{X}, w_{Z}} t \frac{\mathrm{OF}_{X}\left(w_{X}, w_{Z}\right)-\mathrm{OF}_{X}(0,1)}{\mathrm{OF}_{X}(1,0)-\mathrm{OF}_{X}(0,1)}+(1-t) \frac{\mathrm{OF}_{Z}\left(w_{X}, w_{Z}\right)-\mathrm{OF}_{Z}(1,0)}{\mathrm{OF}_{Z}(0,1)-\mathrm{OF}_{Z}(1,0)} \quad \forall t \in[0,1]
$$




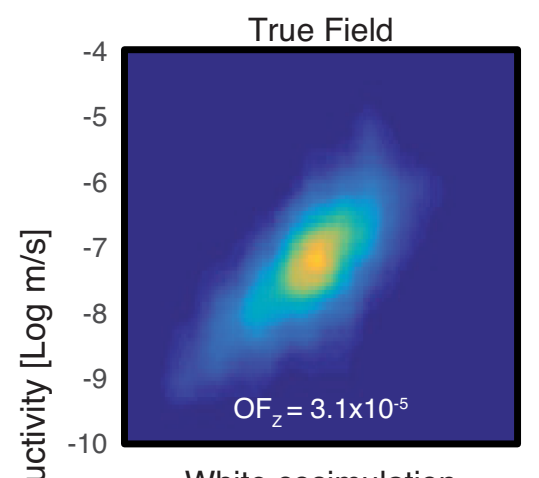

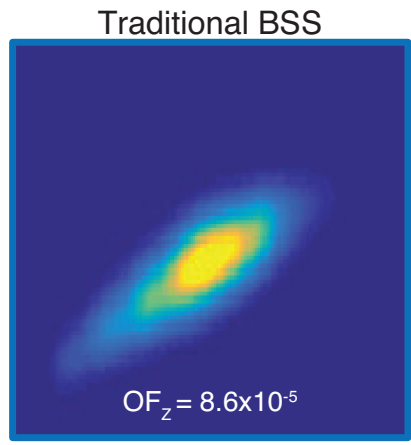

BSS-0.5

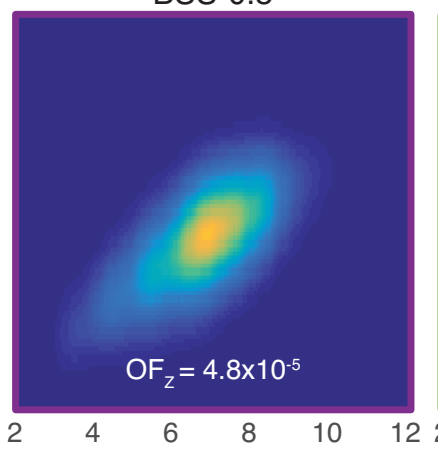

Electrical conductivity $[\mathrm{mS} / \mathrm{m}]$

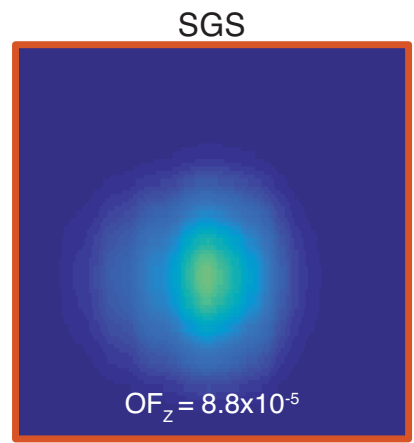

BSS-1

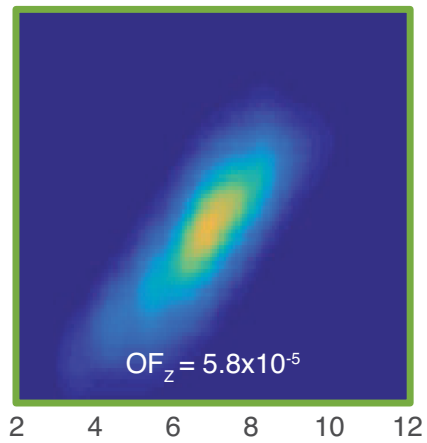

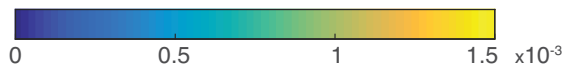

Figure 8. Average joint distributions of 480 realizations for each of the scenarios described in Table 1 together with the joint pdf of the true reference field. The joint pdf of the underlying parametric model is not shown here since it is essentially identical to the true field.

This approach thus allows us to explore various combinations of weights and to make an informed choice based on the importance of reproducing either the relation with the secondary variable or the spatial structure. The minimization of eq. (10) results in six optimal weights combinations (Figs and 11). The corresponding Pareto front, the last point of which corresponds to SGS $\left(w_{X}=1, w_{Z}=0\right)$, can also be visualized in its classical form (Fig. 5).

\subsection{Step-BSS}

Instead of using constant weights throughout simulation process, we now consider an alternative weighting strategy, which involves an abrupt switch from white cosimulation to SGS at some point during the simulation,

$w_{Z}(i)=\left\{\begin{array}{ccc}1 & \text { if } & i<T \\ & 0 & \text { else }\end{array} \quad w_{X}(i)=1-w_{Z}(i)\right.$,

where $0 \leq i \leq 1$ denotes the simulation path index, as quantified by the fraction of simulated grid cells, and $T$ the corresponding threshold index, at which the values of the weights are switched. The motivation for the Step-BSS approach has its origins in the observation that classical BSS fails to adequately emphasize the information of the secondary variable during the initial stages of the simulation process (Fig. 2b) and of the primary variable later on (Fig. 2c). Indeed, at the very beginning, the simulated cells are far apart and define the larger-scale structure of the stochastic realization, such that, at this stage, the information from the secondary variable alone (white cosimulation) is sufficient and appropriate. However, as the simulation progresses, the smoothness imposed by the secondary variable jeopardizes an adequate representation of the fine-scale structure and, hence, the initial dominance of the secondary information should be exchanged in favour of the primary variable (SGS).

The weighting scheme outlined by eq. (11) is tested for different values of the threshold index $T$ ranging from 0 (SGS) to 1 (white cosimulation) with an increment of 0.01 . For each value of $T$, we compute 400 realizations. The resulting objective functions $\mathrm{OF}_{X}$ and $\mathrm{OF}_{Z}$ are shown in light blue in Fig. 5. By changing the value of $T$, Step-BSS explores different optimal solutions depending on the relative importance given to $\mathrm{OF}_{X}(T=0)$ and $\mathrm{OF}_{Z}(T=1)$. Increasing $T$ from 0 to 0.05 , that is, populating the first 5 per cent of the grid exclusively through white cosimulation, dramatically improves the reproduction of the joint pdf, as quantified by $\mathrm{OF}_{Z}$ (Fig. 5). This result can be explained by the fact that the first few simulated cells constrain the larger-scale structures and thus enforce a local correspondence between the simulated primary variable and the smooth secondary variable. In terms of the objective functions, Step-BSS generates slightly better realizations for 


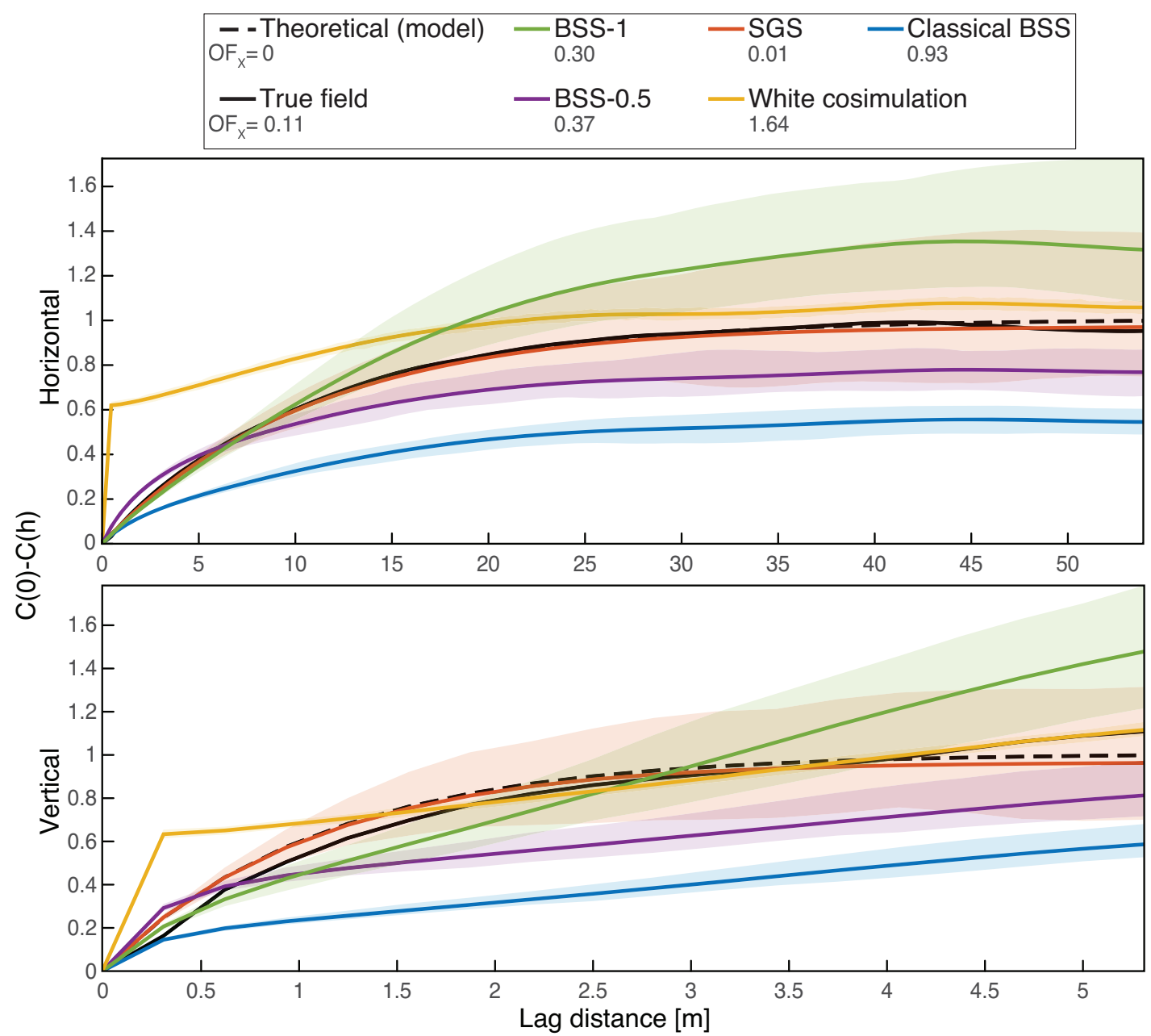

Figure 9. Variograms of the scenarios described in Table 1. The solid coloured lines and corresponding shaded areas denote the averages and the ranges of the variograms of 480 realizations, respectively. The variograms of the true reference field and of the underlying geostatistical model are shown as solid and dotted black lines, respectively.

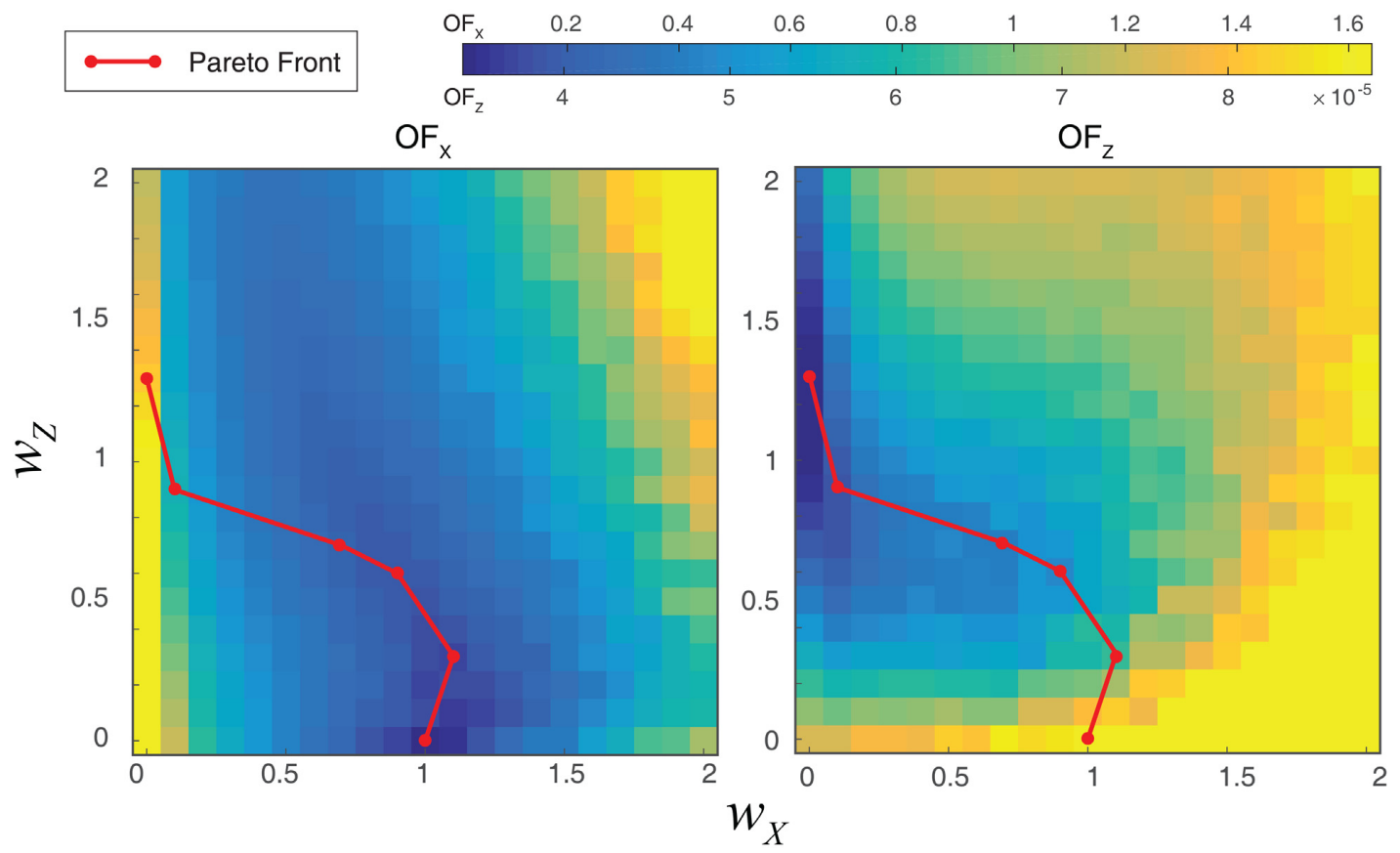

Figure 10. Objective function values as functions of the weights used in the simulations. The colourmap is scaled such that the minima and maxima correspond to the values of the objective functions of SGS and white cosimulation, respectively. 


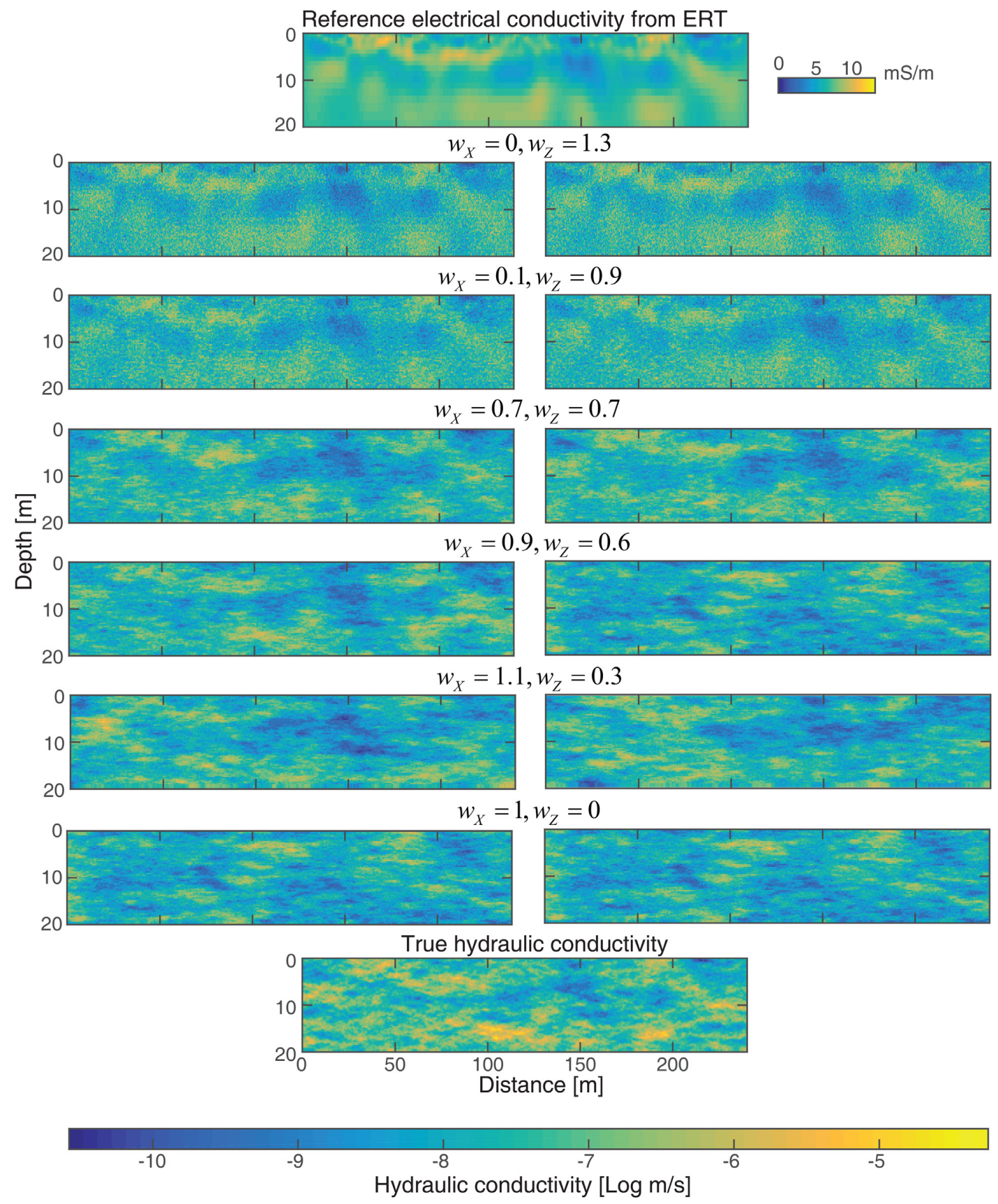

Figure 11. Two realizations for each combination of weights identified along the Pareto front in Fig. 10. The fine-scale structure governed by the primary variable (bottom centre) improves with larger values of $w_{X}$, while larger values of $w_{Z}$ favour the reproduction of the larger-scale structure guided by secondary variable (top centre).

all combinations compared to the other weighting schemes explored so far (Fig. 5). In addition to a step value of 1 in eq. (11), we have also tested other values ranging between 0.9 and 1.3 , which was found to provide very similar results.

\subsection{Multi-step-BSS}

The results of Step-BSS demonstrate that changing the weights during the simulation can be beneficial (Fig. 5). Here, we explore this concept in more detail by considering weights that vary during the entire course of the simulation process. To this end, we divide the simulation path into nine intervals, within which the weights are kept constant. The nine intervals are bounded by eight roughly logarithmically spaced steps located at 1,2,3, 5, 8, 10, 20 and 50 per cent of the simulation path. The parameters of this approach thus consist of the nine weights of $w_{Z}(i)$ used in the corresponding intervals as well as a 10th parameter, which controls the sum of the weights $s=w_{X}(i)+w_{Z}(i)$. The sum of weights remains constant along the simulation, thus allowing to compute $w_{X}(i)$ for each interval as $w_{X}(i)=s-w_{Z}(i)$. Using a Monte-Carlo-type 


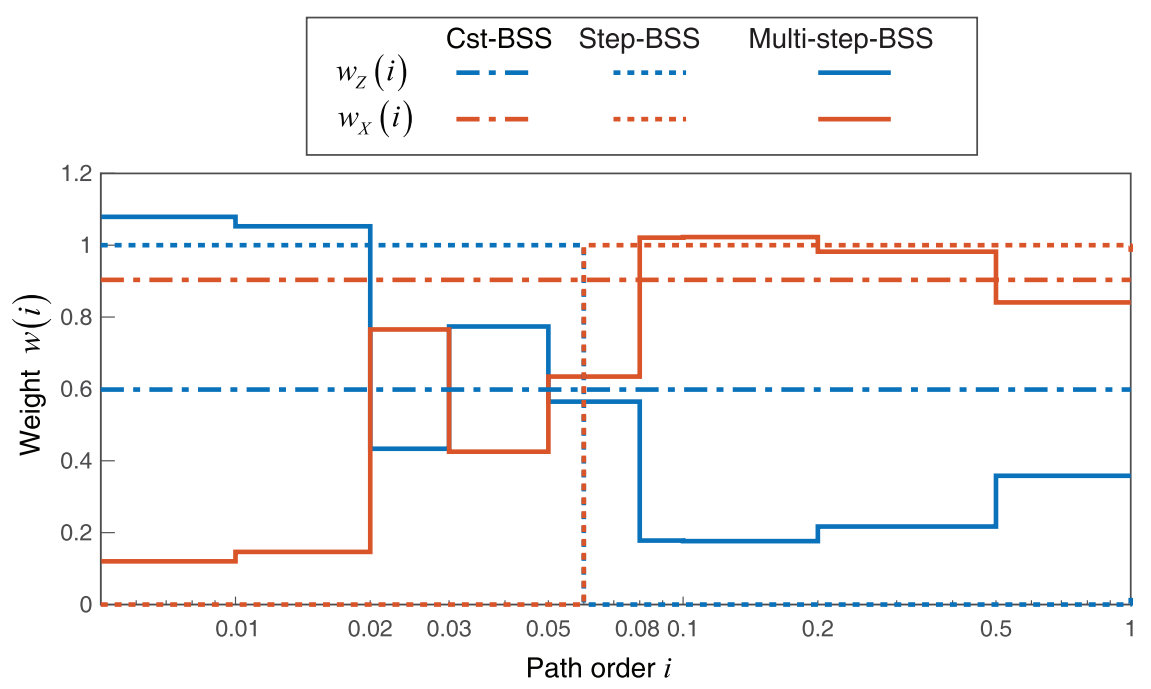

Figure 12. Optimal weights along the simulation path for three weighting schemes: Cst-BSS, Step-BSS and Multi-step-BSS. The log-scale used for the path index $i$ results in roughly equally spaced steps.

approach (Appendix B), a large number of weight combinations are tested to assess the potential benefits of this Multi-step-BSS approach and to explore the optimal parametrization of the associated dynamic weighting scheme (Fig. 12).

The corresponding results are illustrated in the form of two realizations for the optimal Multi-Step-BSS together with the optimal weights used for Step-BSS and the true field (Fig. 13). Multi-step-BSS is capable of reproducing the variogram and the relationship with secondary variable somewhat better than the other weighting schemes considered in this study (Fig. 5). Arguably, the most interesting and pertinent result shown in Fig. 12 is that the optimal sequence of weights bears a striking resemblance to Step-BSS with low and high values of $w_{X}$ and $w_{Z}$, respectively, at the beginning of the simulation, which, following a brief convergence, are then flipped after approximately 8 per cent of the grid has been simulated. A major drawback of Multi-step-BSS is that the rather marginal improvements it provides compared to the other weighting schemes come at a very high computational cost due to the multiple realizations required to optimize the weights.

\section{CONCLUSIONS}

The objective of this study was to improve the BSS-based integration of hydrogeophysical data by accounting for the inherent interdependence between the primary and secondary variables through log-linear pooling. Different from previous computationally expensive and algorithmically complex multistep approaches, this method allows us to perform the data integration process through a single BSS-type simulation step. The method was tested on a synthetic hydrogeophysical data set comprising locally highly resolved, yet spatially sparse, in situ measurements of the hydraulic conductivity and a poorly resolved, yet spatially extensive, ERT-based estimate of the electrical conductivity structure.

Several weighting schemes for the considered log-linear pooling approach were compared by evaluating their ability to reproduce both the spatial statistics (variogram) and the relationship with the secondary variable (joint distribution). First, we demonstrated that simple log-linear pooling with a constant weight of 0.5 (BSS-0.5) or including the marginal distribution with a weight of -1 (BSS-1) already significantly improves the realizations compared to classical BSS. Systematic tests of combinations of two constant weights (Cst-BSS) to explore the trade-offs between either honouring the joint distribution or the variogram allowed us to define a Pareto front, along which a subjective choice with regard to the relative importance of the two criteria can be made. Furthermore, we found that abruptly changing the weights during the simulation (Step-BSS), equivalent to switching from white cosimulation to SGS, in the very early stages of the simulation process provided even better results than using constant weights. Finally, tests with a Multi-step-BSS, where the weights can change dynamically in the course of the simulation process, confirmed the key characteristics observed for Step-BSS: the larger-scale structure (secondary variable) should be accounted for at the very beginning of the simulation process followed by strong emphasis on the smaller-scale fluctuations (primary variable). This approach allowed for additional minor improvements, albeit at the price of a complex parametrization and a costly Monte-Carlo-type search procedure for determining the optimal sequence of weights along the simulation path.

The results of this study demonstrate that it is possible to reduce the redundancy of information inherent to classical BSS with a flexible $\log$-linear-pooling-based weighting scheme. Yet, generalizations of any quantitative aspects are difficult because of the wide variability of possible scenarios, such as differences in resolution between the primary and the secondary variables, the form of the joint distribution, the correspondence between the variograms of the primary and the secondary variables, or the location and quantity of hard data. Nevertheless, it is possible to provide some fundamental recommendations. From a practical point of view, our results clearly indicate that is advisable to use simple calibrated weighting schemes, such as Step-BSS or Cst-BSS, as they provide arguably the best balance between the quality of the realizations and the associated computational cost. With regard to non-calibrated weighting schemes, our study demonstrates that classical BSS should be avoided in preference of basic log-linear pooling schemes, such as BSS-0.5 or BSS-1. 

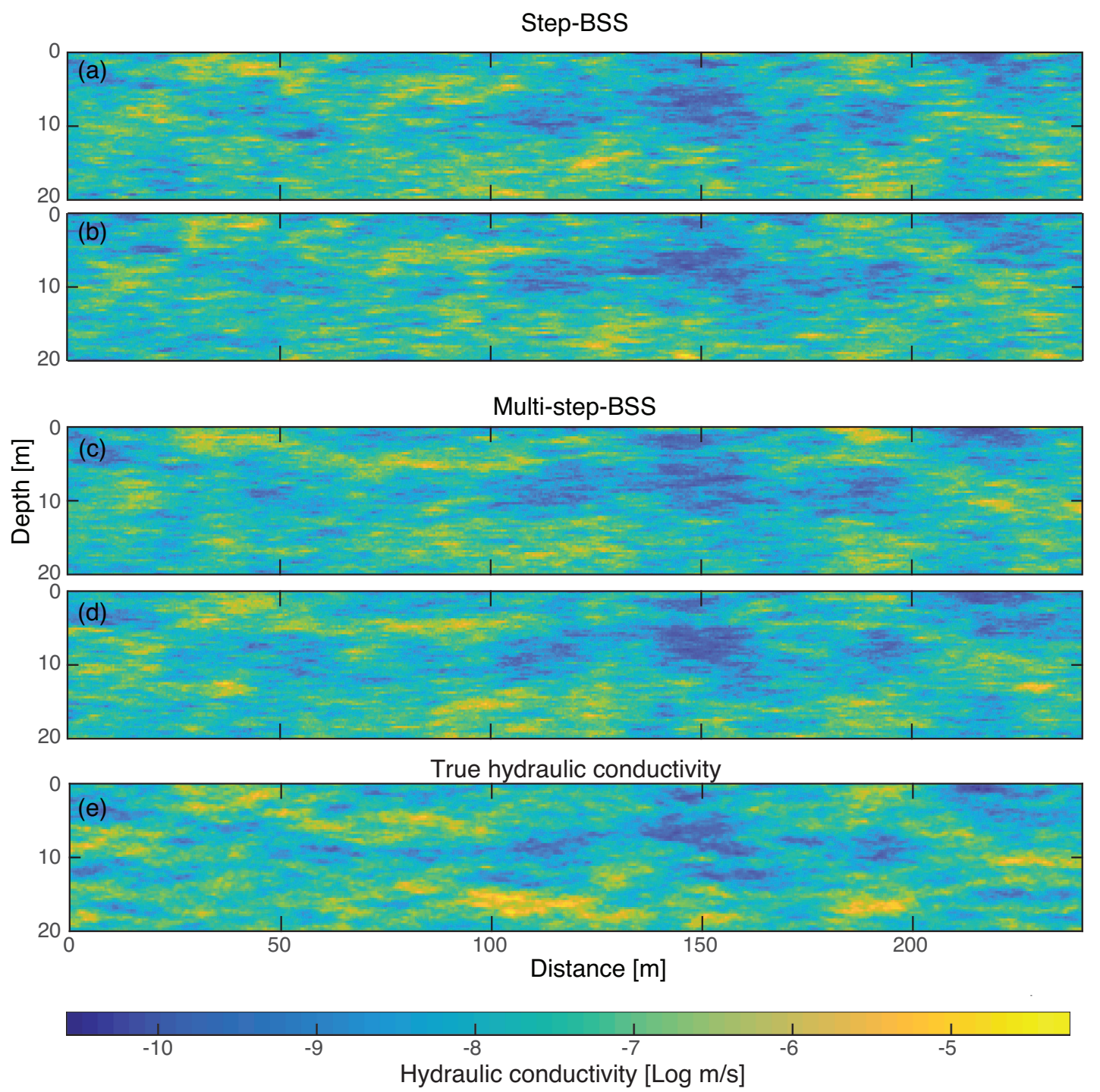

Figure 13. Comparison of two realizations of the optimal (a,b) Step-BSS and (c,d) Multi-step-BSS weighting schemes together with (e) the true reference field of hydraulic conductivity.

\section{ACKNOWLEDGEMENTS}

This work has been supported by grant \#200021_152917 from the Swiss National Science Foundation. We thank Thomas Mejer Hansen, Klaus Mosegaard and three anonymous referees as well as the editor Ute Weckmann for their efforts. Lucid comments and suggestions by Denis Allard, Lionel Benoit and the editor-in-chief Jörg Renner helped to improve the quality of this manuscript and are gratefully acknowledged. We also acknowledge LIASHyC (https://www.liashyc.org) for travel support.

\section{REFERENCES}

Allard, D., Comunian, A. \& Renard, P., 2012. Probability aggregation methods in geoscience, Math. Geosci., 44(5), 545-581.

Archie, G.E., 1942. The electrical resistivity $\log$ as an aid in determining some reservoir characteristics, Trans. AIME, 146(01), $54-62$.

Binley, A. \& Kemna, A., 2005. DC resistivity and induced polarization methods, in Hydrogeophysics, Vol. 50, pp. 129-156, eds Rubin, Y. \& Hubbard, S.S., Springer Netherlands, doi:10.1007/1-4020-3102-5_5.

Chen, J., Hubbard, S. \& Rubin, Y., 2001. Estimating the hydraulic conductivity at the South Oyster Site from geophysical tomographic data using Bayesian techniques based on the normal linear regression model, Water Resour. Res., 37(6), 1603-1613.

Chilès, J.-P. \& Delfiner, P., 1999. Geostatistics, Vol. 497, John Wiley \& Sons, Inc.
Comunian, A., Renard, P. \& Straubhaar, J., 2012. 3D multiple-point statistics simulation using 2D training images, Comput. Geosci., 40, 49-65, doi:10.1016/j.cageo.2011.07.009.

Dafflon, B., Irving, J. \& Holliger, K., 2009. Simulated-annealing-based conditional simulation for the local-scale characterization of heterogeneous aquifers, J. Appl. Geophys., 68(1), 60-70.

Deutsch, C.V. \& Journel, A.G., 1992. GSLIB: Geostatistical Software Library and User's Guide, Oxford University Press.

Deutsch, C.V. \& Wen, X.H., 2000. Integrating large-scale soft data by simulated annealing and probability constraints, Math. Geol., 32(1), 49-67.

Doligez, B., Le Ravalec-Dupin, M., Bouquet, S. \& Adelinet, M., 2015. A review of three geostatistical techniques for realistic geological reservoir modeling integrating multi-scale data, Bull. Can. Pet. Geol., 63(4), 277-286. 
Doyen, P.M. \& Boer, L.D., 1996. Bayesian sequential Gaussian simulation of lithology with non-linear data, U.S. Patent 5539704 A.

Doyen, P.M., den Boer, L.D. \& Pillet, W.R., 1996. Seismic porosity mapping in the Ekofisk Field using a new form of collocated cokriging, In $S E G$ Technical Program Expanded Abstracts 1996, pp. 1931-1931, Society of Exploration Geophysicists, doi:10.2118/36498-MS.

Dubreuil-Boisclair, C., Gloaguen, E., Marcotte, D. \& Giroux, B., 2011. Heterogeneous aquifer characterization from ground-penetrating radar tomography and borehole hydrogeophysical data using nonlinear Bayesian simulations, Geophysics, 76(4), J13-J25.

Emery, X., 2004. Testing the correctness of the sequential algorithm for simulating Gaussian random fields, Stoch. Environ. Res. Risk Assess., 18(6), 401-413.

Ezzedine, S., Rubin, Y. \& Chen, J., 1999. Bayesian method for hydrogeological site characterization using borehole and geophysical survey data: theory and application to the Lawrence Livermore National Laboratory Superfund Site, Water Resour. Res., 35(9), 2671.

Genest, C. \& Zidek, J.V., 1986. Combining probability distributions: a critique and an annotated bibliography, Stat. Sci., 1(1), 147-148.

Gómez-Hernández, J.J. \& Journel, A.G., 1993. Joint sequential simulation of multigaussian fields, in Geostatistics Tróia '92, pp. 85-94, ed. Soares, A., Springer Netherlands, doi:10.1007/978-94-011-1739-5_8.

Gómez-Hernández, J.J. \& Wen, X.-H., 1998. To be or not to be multiGaussian? A reflection on stochastic hydrogeology, Adv. Water Res., 21(1), $47-61$.

Guardiano, F.B. \& Srivastava, R.M., 1993. Multivariate geostatistics: beyond bivariate moments, in Geostatistics Tròia '92, pp. 133-144, ed. Soares, A., Springer Netherlands.

Heinz, J., Kleineidam, S., Teutsch, G. \& Aigner, T., 2003. Heterogeneity patterns of Quaternary glaciofluvial gravel bodies (SWGermany): application to hydrogeology, Sediment. Geol., 158(1-2), 1-23, doi:10.1016/S0037-0738(02)00239-7.

Hoffimann, J., Scheidt, C., Barfod, A. \& Caers, J., 2017. Stochastic simulation by image quilting of process-based geological models, Comput. Geosci., 106(February), 18-32, doi:10.1016/j.cageo.2017.05.012.

Hu, L.Y., Le Ravalec-Dupin, M. \& Blanc, G., 2001. Gradual deformation and iterative calibration of truncated Gaussian simulations, Pet. Geosci., 7(S), S25-S30.

Hyndman, D.W. \& Gorelick, S.M., 1996. Estimating lithologic and transport properties in three dimensions using seismic and tracer data: the Kesterson aquifer, Water Resour. Res., 32(9), 2659-2670.

Journel, A.G., 1974. Geostatistics for conditional simulation of ore bodies, Econ. Geol., 69(5), 673-687.

Journel, A.G., 1989. Fundamentals of Geostatistics in Five Lessons, Vol. 16, American Geophysical Union.
Journel, A.G., 1999. Markov models for cross-covariances, Math. Geol., 31(8), 955-964.

Le Ravalec-Dupin, M., Noetinger, B. \& Hu, L.Y., 2000. The FFT moving average (FFT-MA) generator: an efficient numerical method for generating and conditioning Gaussian simulations, Math. Geol., 32(6), 701-723, doi:10.1023/A:1007542406333.

Mariethoz, G., Renard, P., Cornaton, F. \& Jaquet, O., 2009a. Truncated plurigaussian simulations to characterize aquifer heterogeneity, Ground Water, 47(1), 13-24.

Mariethoz, G., Renard, P. \& Froidevaux, R., 2009b. Integrating collocated auxiliary parameters in geostatistical simulations using joint probability distributions and probability aggregation, Water Resour. Res., 45(8), doi:10.1029/2008WR007408.

Nussbaumer, R., Mariethoz, G., Gravey, M., Gloaguen, E. \& Holliger, K., 2018. Accelerating sequential gaussian simulation with a constant path, Comput. Geosci., 112(2018), 121-132, doi:10.1016/j.cageo.2017.12.006.

Rivoirard, J., 2001. Which models for collocated cokriging? Math. Geol., 33(2), 117-131.

Rivoirard, J., 2004. On some simplifications of cokriging neighborhood, Math. Geol., 36(8), 899-915.

Rubin, Y. \& Hubbard, S. 2005. Stochastic forward and inverse modeling: the "hydrogeophysical" challenge, in Hydrogeophysics, pp. 487-511, Springer Netherlands, doi:10.1007/1-4020-3102-5_17.

Ruggeri, P., Irving, J., Gloaguen, E. \& Holliger, K., 2013. Regional-scale integration of multiresolution hydrological and geophysical data using a two-step Bayesian sequential simulation approach, Geophys. J. Int., 194(1), 289-303.

Ruggeri, P., Gloaguen, E., Lefebvre, R., Irving, J. \& Holliger, K., 2014. Integration of hydrological and geophysical data beyond the local scale: application of Bayesian sequential simulation to field data from the Saint-Lambert-de-Lauzon site, Québec, Canada, J. Hydrol., 514, 271-280.

Tarantola, A., 2005. Inverse Problem Theory and Methods for Model Parameter Estimation, Society for Industrial and Applied Mathematics.

Verly, G.W., 1993. Sequential gaussian cosimulation: a simulation method integrating several types of information, in Geostatistics Tròia '92, pp. 543-554, ed. Soares, A., Kluwer Academic Publishers, doi:10.1007/97894-011-1739-5_42.

Xu, W., Tran, T.T., Srivastava, R.M. \& Journel, A.G., 1992. Integrating seismic data in reservoir modeling: the collocated cokriging alternative, in Proceedings of SPE Annual Technical Conference and Exhibition, pp. 833-842, Society of Petroleum Engineers, doi.org/10.2118/24742-MS.

Zinn, B. \& Harvey, C.F., 2003. When good statistical models of aquifer heterogeneity go bad: a comparison of flow, dispersion, and mass transfer in connected and multivariate Gaussian hydraulic conductivity fields, Water Resour. Res., 39(3), 1-19, doi:10.1029/2001WR001146.

\section{APPENDIX A}

In the following, we show that, because of its sequential nature, BSS inherently relies on the assumption of conditional independence between the secondary variable and the previously simulated values of the primary variable.

Let us consider the conditional probability of an unknown event $A$ based on several known events $\left\{D_{1}, \ldots, D_{n}\right\}$

$P\left(A \mid D_{1}, \ldots, D_{n}\right)=\frac{P\left(A, D_{1}, \ldots, D_{n}\right)}{P\left(D_{1}, \ldots, D_{n}\right)}$

Substituting the numerator of eq. (A1) by the chain rule of probability

$P\left(A_{1}, \ldots A_{n}\right)=\prod_{k=1}^{n} P\left(A_{k} \mid A_{1}, \ldots A_{k-1}\right)$,

leads to

$P\left(A \mid D_{1}, \ldots, D_{n}\right)=P(A) \frac{\prod_{j=1}^{n} P\left(D_{j} \mid A, D_{1}, \ldots, D_{j-1}\right)}{P\left(D_{1}, \ldots, D_{n}\right)}$. 
In the context of sequential simulation, $A$ represents the simulated variable $X_{i}$ and the conditional data $D$ is composed of the previously simulated cells $X_{<i}=\left\{X_{1}, \ldots, X_{i-1}\right\}$

$P\left(X_{i} \mid X_{<i}\right)=\frac{P\left(X_{i}\right) \prod_{j=1}^{i-1} P\left(X_{j} \mid X_{i}, X_{<j}\right)}{P\left(X_{<i}\right)}$.

Yet, in BSS, $D$ also includes the collocated secondary variable $Z_{i}$, which leads to

$P\left(X_{i} \mid X_{<i}, Z_{i}\right)=\frac{P\left(X_{i}\right) \prod_{j=1}^{i-1} P\left(X_{j} \mid X_{i}, X_{<j}\right)}{P\left(X_{<i}, Z_{i}\right)} P\left(Z_{i} \mid X_{i}, X_{<i}\right)$.

Combining eqs (A4) and (A5) yields

$P\left(X_{i} \mid X_{<i}, Z_{i}\right)=\frac{P\left(X_{i} \mid X_{<i}\right) P\left(X_{<i}\right)}{P\left(X_{<i}, Z_{i}\right)} P\left(Z_{i} \mid X_{i}, X_{<i}\right)$.

As $P\left(X_{<i}\right)$ and $P\left(X_{<i}, Z_{i}\right)$ are constant, eq. (A6) can be reduced to the proportionality

$P\left(X_{i} \mid X_{<i}, Z_{i}\right) \propto P\left(X_{i} \mid X_{<i}\right) P\left(Z_{i} \mid X_{i}, X_{<i}\right)$.

\section{APPENDIX B}

Here, we describe the algorithmic details of the Metropolis-Hastings search we performed to determine the optimal sequence of weights along the simulation path for our analysis of the Multi-step-BSS approach.

1. Initialization. Of a total of ten parameters, the first nine define the value of $w_{Z}(i)$ for the corresponding nine intervals along the simulation path, while the last parameter controls to the sum of the weights $s=w_{X}(i)+w_{Z}(i)$ which is constant for all intervals and, hence, $w_{X}(i)=s-w_{Z}(i)$. The initial parameters are selected to reproduce the optimal Step-BSS, that is, $\mathbf{m}_{0}=\left[\begin{array}{llllllllll}1 & 1 & 1 & 1 & 1 & 0 & 0 & 0 & 0 & 1\end{array}\right]$.

2. For each iteration $i$

(i) Generate a new set of model parameters $\mathbf{m}_{i}$. The proposal distribution is a Gaussian function with a variance of 0.05 , that is, $\mathbf{m}_{i}=\mathbf{m}_{i-1}+\mathcal{N}(\mathbf{0}, 0.05)$. The sum of the weights $\mathbf{m}(10)$ is not constrained and can evolve freely. The other nine parameters can vary between 0 and the sum of the weights. If a parameter is proposed outside these bounds, it is reflected on the bound, that is, the value of the parameter $\mathbf{m}_{i}$ becomes $b-\left(\mathbf{m}_{i}-b\right)$, with $b$ being the corresponding bound value.

(ii) Calculate the acceptance ratio. The likelihood function is

$P\left(\mathbf{m}_{i}\right)=\exp \left\{-O F\left(\mathbf{m}_{i}\right) / \sigma\right\}$,

where $O F(\mathbf{m})$ is the normalized sum of objective functions defined in eq. (10) with $t=0.5$ such that equal importance is given to each objective function and $\sigma=0.02$ corresponds to the errors in determining the objective function. The value of $\sigma$ is manually calibrated such that the acceptance ratio of the Metropolis-Hastings algorithm, which is described in the next step, is around 40 per cent for faster convergence. The acceptance ratio is therefore $\alpha=P\left(\mathbf{m}_{i}\right) / P\left(\mathbf{m}_{i-1}\right)$.

(iii) Acceptance. The acceptance is defined based on a uniform random number $u \sim \mathcal{U}(0,1)$. If $u<\alpha$, the proposed set of parameters $\mathbf{m}_{i}$ is accepted, and otherwise rejected so that $\mathbf{m}_{i}=\mathbf{m}_{i-1}$.

The Metropolis-Hastings search was stopped after 6000 iterations with an acceptance rate of 51 per cent. As the initial parameter set corresponds to the optimized parameters of step-BSS, no burn-in was considered. 